\title{
Integrated analysis of hydrothermal flow through pretreatment
}

Veronique Archambault-Leger ${ }^{1}$, Xiongjun Shao ${ }^{1}$ and Lee R Lynd ${ }^{1,2,3^{*}}$

\begin{abstract}
Background: The impact of hydrothermal flowthrough (FT) pretreatment severity on pretreatment and solubilization performance metrics was evaluated for three milled feedstocks (corn stover, bagasse, and poplar) and two conversion systems (simultaneous saccharification and fermentation using yeast and fungal cellulase, and fermentation by Clostridium thermocellum).

Results: Compared to batch pretreatment, FT pretreatment consistently resulted in higher XMG recovery, higher removal of non-carbohydrate carbon and higher glucan solubilization by simultaneous saccharification and fermentation (SSF). XMG recovery was above 90\% for FT pretreatment below 4.1 severity but decreased at higher severities, particularly for bagasse. Removal of non-carbohydrate carbon during FT pretreatment increased from 65\% at low severity to $80 \%$ at high severity for corn stover, and from $40 \%$ to $70 \%$ for bagasse and poplar.

Solids obtained by FT pretreatment were amenable to high conversion for all of the feedstocks and conversion systems examined. The optimal time and temperature for FT pretreatment on poplar were found to be 16 min and $210^{\circ} \mathrm{C}$. At these conditions, SSF glucan conversion was about $85 \%, 94 \%$ of the XMG was removed, and $62 \%$ of the non carbohydrate mass was solubilized. Solubilization of FT-pretreated poplar was compared for $C$. thermocellum fermentation (10\% inoculum), and for yeast-fungal cellulase SSF (5\% inoculum, cellulase loading of 5 and 10 FPU/g glucan supplemented with $\beta$-glucosidase at 15 and $30 \mathrm{U} / \mathrm{g}$ glucan). Under the conditions tested, which featured low solids concentration, C. thermocellum fermentation achieved faster rates and more complete conversion of FT-pretreated poplar than did SSF. Compared to SSF, solubilization by C. thermocellum was 30\% higher after 4 days, and was over twice as fast on ball-milled FT-pretreated poplar.

Conclusions: XMG removal trends were similar between feedstocks whereas glucan conversion trends were significantly different, suggesting that factors in addition to XMG removal impact amenability of glucan to enzymatic attack. Corn stover exhibited higher hydrolysis yields than bagasse or poplar, which could be due to higher removal of non-carbohydrate carbon. XMG in bagasse is more easily degraded than XMG in corn stover and poplar. Conversion of FT-pretreated substrates at low concentration was faster and more complete for C. thermocellum than for SSF.
\end{abstract}

Keywords: Flowthrough pretreatment, Hydrothermal pretreatment, Biofuel, Cellulosic, Xylan, SSF, Consolidated bioprocessing, Clostridium thermocellum

\footnotetext{
* Correspondence: Lee.R.Lynd@Dartmouth.edu

'Dartmouth College, Hanover, NH 03755, USA

${ }^{2}$ DOE BioEnergy Science Center, Oak Ridge National Laboratory, Oak Ridge,

TN 37831, USA

Full list of author information is available at the end of the article
} 


\section{Background}

Producing fuel from lignocellulosic biomass is of interest in light of pressing concerns about petroleum supply and climate change [1-4]. The main obstacle impeding production of cost-competitive cellulosic biofuels is the high cost of converting cellulosic feedstocks to reactive intermediates, termed biomass recalcitrance. In the case of biological conversion of cellulosic biomass to sugars, recalcitrance results from incomplete accessibility of attack by microbes and their saccharolytic enzymes due to structural features, heterogeneous composition, and chemical linkages between these components $[5,6]$.

In the biomass conversion field, "pretreatment" refers to the process step that converts cellulosic biomass into a form amenable to biological attack. Various approaches to pretreatment allow hydrolysis yields of $90 \%$ or more, whereas low yields have been widely observed in the absence of pretreatment $[7,8]$. Pretreatment processes examined in the literature include exposure to acid or alkali, ammonia, lime, organic solvents, ionic liquids, and water, generally at elevated temperature and pressure [7-10]. Once cellulosic biomass is rendered amenable to biological attack, there are different approaches to ferment the substrate. In simultaneous saccharification and fermentation (SSF), cellulose hydrolysis and hexose fermentation occur in a separate unit operation from cellulase production. This configuration has several advantages, but the cost of cellulase remains a significant barrier [11-13]. In consolidated bioprocessing (CBP), cellulase production, cellulose hydrolysis, as well as hexose and pentose fermentations are all achieved in one process. CBP is in principle attractive because of streamlined processing and no costs for added enzymes, however development of requisite microorganisms is a work in progress.

Pretreatment has multiple objectives that are challenging to achieve at once. In particular, high cellulose reactivity is fostered by reaction at high temperature and long reaction times, yet such conditions commonly result in degradation of sugars and production of fermentation inhibitors. Addition of chemicals allows reactive solids to be obtained at lower temperatures and shorter times than would otherwise be possible, but involves costs due to purchase and/or recycle of the chemicals. Pretreatment accounts for a substantial fraction of the cost of processing biomass [14-16], has pervasive impacts on the performance and thus cost of hydrolysis and fermentation $[9,14,17]$, and improvements in pretreatment are widely recognized as a key route to improving the costcompetitiveness of biomass conversion $[10,18]$.

In addition to liquid phase composition, temperature and residence time, the configuration of pretreatment processes is also an important factor impacting performance. In particular, operation of pretreatment in a flow through (FT) configuration has been proposed and investigated to some degree, generally with a water or dilute acid liquid phase [14,19-22]. In FT pretreatment, the ratio of liquid and solid residence times, $R_{L / S}$, is less than one, whereas in the absence of flow through whether the process is operated in batch or continuous mode $-R_{L / S}$ is equal to unity. As a result of liquid being removed from the reactor, solubilized sugars have less time to degrade, and recondensation of solubilized lignin and xylan on cellulose fibers upon cooling occurs to a lesser extent [14,21]. Consistent with this understanding, FT pretreatment typically achieves higher solids reactivity, higher xylan removal, less sugar degradation and substantially higher removal of lignin and other noncarbohydrate carbon compared to pretreatment in non flow through configurations at the same temperature and residence time $[5,7-10,14]$. The relationship between lignin removal and xylan removal is nearly linear, and it has been suggested that lignin and xylan are removed as complexes and that lignin disruption is a key determinant of solids digestibility [14,22]. Other studies have looked at the mass ratio of liquid to solids $[23,24]$ or flow velocity $[19,20,25]$, indicating that fluid flow has an impact on pretreatment mechanism whereas dilution effects may not be significant below $10 \mathrm{w} / \mathrm{v} \%$. To realize the advantages of FT pretreatment in a practical context, it is necessary to address the mechanical complexities of arranging a bed of biomass for flow through configuration at scale while also avoiding unacceptably high energy requirements and sugar dilution.

While we are optimistic that such realization is possible, this will require integrated understanding of fluid mechanics, kinetics, heat and mass transfer, and how these are impacted by feedstock properties, operating conditions, and the choice of conversion system. In order to provide a foundation for such studies, we undertake here to evaluate performance metrics for FT pretreatment as a function of time and temperature for corn stover, sugar cane bagasse and poplar, and also to compare conversion of FT-pretreated poplar by SSF and by C. thermocellum, a candidate CBP organism [11]. We are not aware of a prior study that has evaluated FT pretreatment for such a range of feedstocks and conversion systems.

\section{Results and discussion}

\section{Effect of poplar moisture content}

A control experiment was performed to study whether feedstock moisture impacted sugar recovery and enzymatic digestibility. Wet and dry milled poplar were FT pretreated at $180^{\circ} \mathrm{C}$ for $8 \mathrm{~min}$ (ratio of liquid and solid residence times, $\left.\mathrm{R}_{\mathrm{L} / \mathrm{S}}=0.25\right)$ and at $200^{\circ} \mathrm{C}$ for $16 \min \left(\mathrm{R}_{\mathrm{L} /}\right.$ $\mathrm{s}=0.125$ ), and xylan/mannan/galactan (XMG) recovery and solubilization along with glucan conversion after SSF were analyzed. SSF results were based on yeast fermentation (Saccharomyces cerevisae strain D5A) prepared in 
YPD medium supplemented with $16.7 \mathrm{mg}$ cellulase/g glucan (10 FPU/g glucan) and $30 \mathrm{IU} / g$ glucan Novozyme $\beta$ glucosidase. As shown in Figure 1, glucan conversion, XMG recovery and XMG solubilization appear to be higher for dried substrate than wet substrate, although the difference is not statistically significant. The standard error, estimated from the triplicate of a separate experiment, was $1.9 \%, 1.5 \%$ and $1.3 \%$ for glucan conversion, XMG recovery and XMG solubilization respectively. Since dry poplar sample is easier to mill and store, it was used for further analysis.

\section{Initial batch and FT pretreatments}

Milled corn stover, sugar cane bagasse and poplar were FT pretreated for $12 \mathrm{~min}$ at $220^{\circ} \mathrm{C}\left(\mathrm{R}_{\mathrm{L} / \mathrm{S}}=0.167\right)$ and batch pretreated for $14 \mathrm{~min}$ at $220^{\circ} \mathrm{C}\left(\mathrm{R}_{\mathrm{L} / \mathrm{S}}=1,22.5 \mathrm{w} / \mathrm{v} \%\right)$ to allow for the greater heat-up time in batch (see methods). After $96 \mathrm{~h}$ of SSF at the conditions specified above, glucan conversion was $93 \%$ for corn stover, $90 \%$ for bagasse, and $79 \%$ for poplar whereas batch pretreatment allowed about $75 \%$ glucan conversion for corn stover, $68 \%$ for bagasse and $50 \%$ for poplar (Figure 2A). Recovery of glucan and XMG fractions was evaluated based on the percent present in all forms (insoluble, oligomer, monomer) at the end of the experiment relative to that at the start of the experiment. On this basis, glucan recovery was $95-100 \%$ for batch and FT pretreatments on all substrates. XMG recovery ranged from $69 \%$ to $84 \%$ for batch pretreatment and from $84 \%$ to $92 \%$ for FT pretreatment, as shown in Figure 2B. Extraction of non-carbohydrate carbon (Figure 2C), mostly lignin, was modest for batch (30\% for bagasse, $38 \%$ for poplar and $46 \%$ for corn stover) but much more pronounced for FT pretreatment (58\% for bagasse, $68 \%$ for poplar and $78 \%$ for corn stover). The higher solids reactivity, XMG recovery and removal of non carbohydrate carbon for FT pretreatment compared to batch observed in this study are consistent with results obtained by Liu and Wyman [20] and Yang and Wyman [14,23] for corn stover. This study showed that the higher solids reactivity, XMG recovery and removal of non carbohydrate carbon for FT pretreatment compared to batch were also observed with bagasse and poplar. For both FT and batch pretreatments, poplar's conversion is lower and its XMG recovery higher compared with corn stover and bagasse. Moreover, the removal of non carbohydrate carbon is higher in corn stover than in the other substrates studied.

\section{Exploration of time and temperature for FT pretreatment}

Data trends for milled poplar, bagasse and corn stover were visualized by plotting glucan conversion, removal of XMG and non-carbohydrate carbon, and XMG recovery as a function of severity, defined as $\log R o=\log \left(t \frac{\exp ^{T-100}}{14.75}\right) \quad[15]$, where $\mathrm{t}$ is the time of reaction (minutes) and $\mathrm{T}$ is the temperature of the reaction $\left({ }^{\circ} \mathrm{C}\right)$. A range of severities from 3.2 to 5.1 was obtained by varying the time of pretreatment from 8 to $24 \mathrm{~min}\left(\mathrm{R}_{\mathrm{L} / \mathrm{S}}=0.250-0.083\right)$ and the temperature from $180^{\circ} \mathrm{C}$ to $225^{\circ} \mathrm{C}$.

\section{Glucan conversion}

The conversion of glucan in pretreated biomass during SSF is a direct measure of the digestibility of the substrate. It was found, as illustrated in Figure 3A, that the conversion follows a second order polynomial fit with similar shape for corn stover, bagasse and poplar. The biomass is increasingly digestible as time and temperature increase until an optimum point is reached, beyond which glucan conversion decreases. The optimum point varies slightly between substrates, likely due to their differences in composition and structure. The optimal severity was 4.1 for corn stover $\left(16 \mathrm{~min}, 200^{\circ} \mathrm{C}, \mathrm{R}_{\mathrm{L} / \mathrm{S}}=0.125\right)$, 4.4 for poplar $\left(16 \mathrm{~min}, 210^{\circ} \mathrm{C}, \mathrm{R}_{\mathrm{L} / \mathrm{S}}=0.125\right)$, and 4.6 for bagasse (12 $\mathrm{min}$, $\left.220^{\circ} \mathrm{C}, \mathrm{R}_{\mathrm{L} / \mathrm{S}}=0.167\right)$. The higher glucan conversion for corn stover at a lower severity is likely due to the higher non-carbohydrate removal compared to other substrates discussed below.

\section{XMG removal/recovery}

XMG removal followed similar trends for the different feedstocks tested, although slightly lower values were

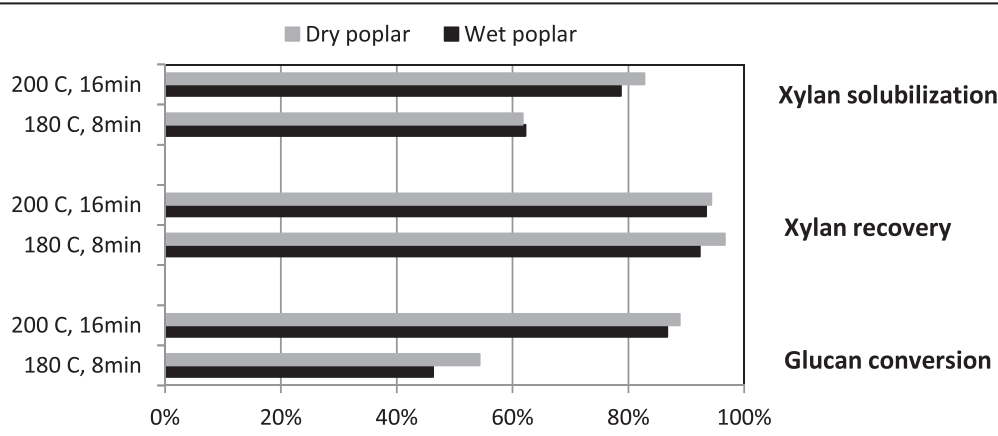

Figure 1 Comparison between dry and wet poplar pretreated at two different sets of time and temperature. 


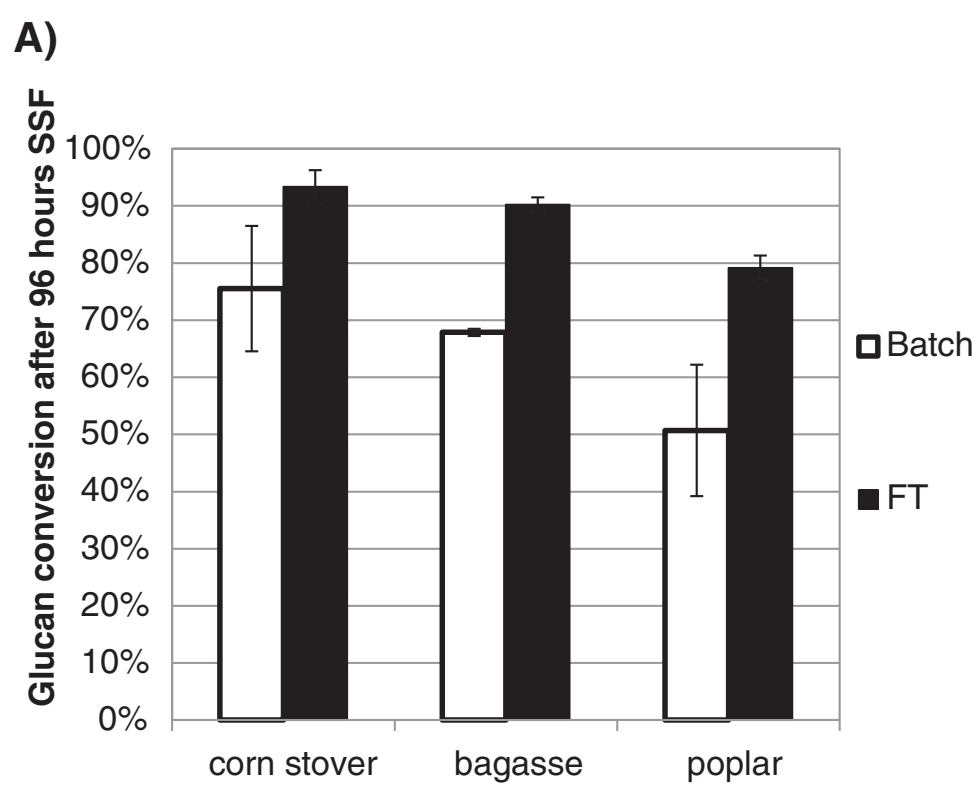

\section{B)}
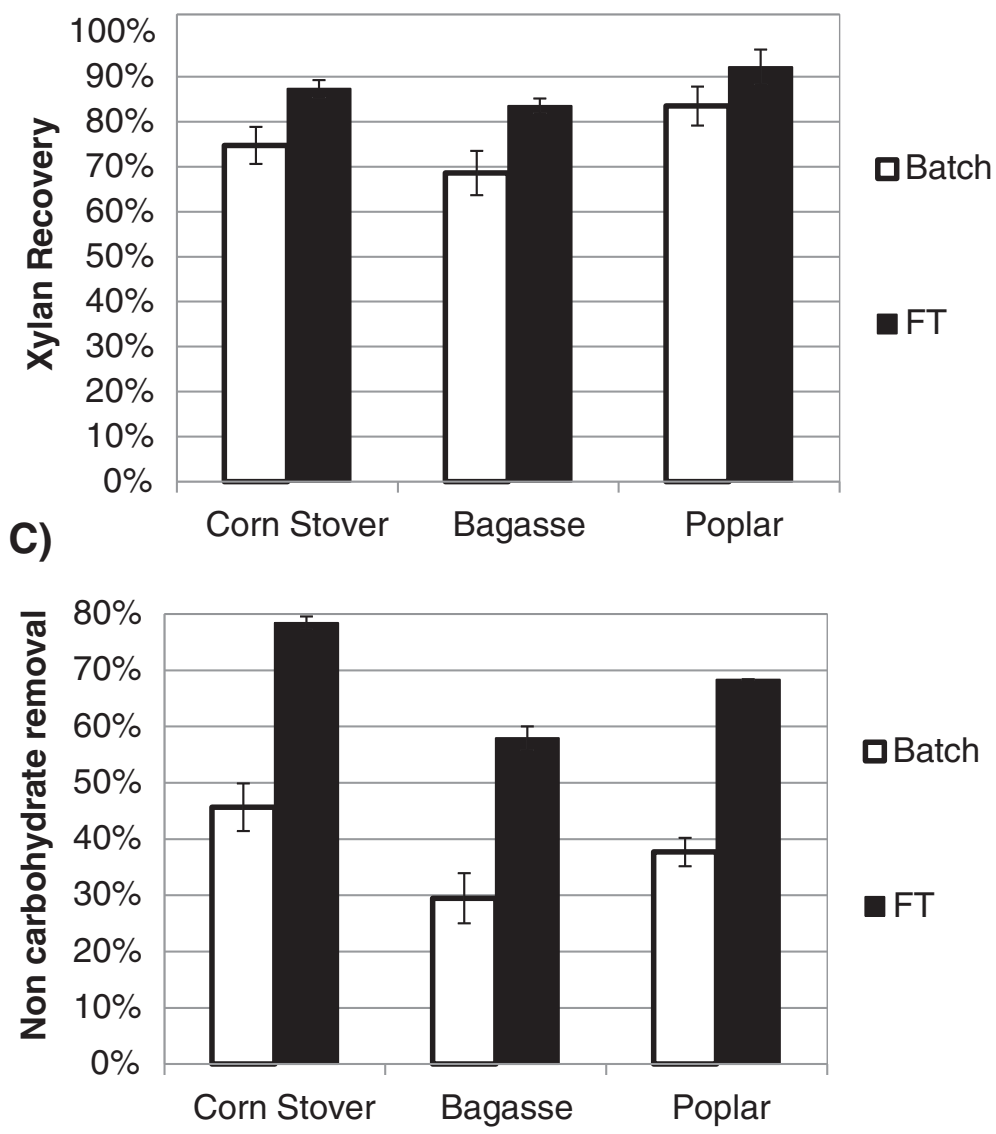

Figure 2 Batch and FT pretreatment comparison for pretreated corn stover, poplar and bagasse. A) glucan conversion, B) sugar recovery and C) extraction of non-carbohydrate carbon. Pretreatment Conditions: $220^{\circ} \mathrm{C}, 12 \mathrm{~min}$ (14 min for batch pretreatment), flow rate: $30 \mathrm{~mL} / \mathrm{min}$, particle size: 2 mm. Enzyme loading for SSF: 10 FPU/g glucan (11.7 mg enzymes/g solids). Initial substrate concentration: 20 g/L glucan. The error bars show one standard deviation on duplicates. 

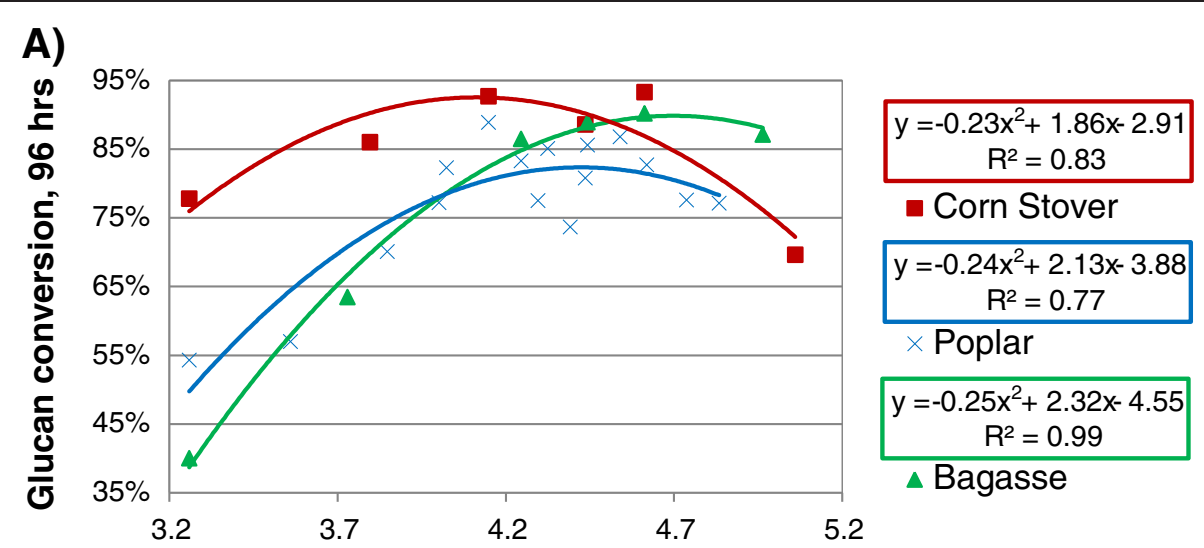

$$
\begin{array}{|c|}
\hline y=-0.24 x^{2}+2.13 x-3.88 \\
R^{2}=0.77 \\
x \text { Poplar }
\end{array}
$$

$$
\begin{gathered}
y=-0.25 x^{2}+2.32 x-4.55 \\
R^{2}=0.99
\end{gathered}
$$

$\triangle$ Bagasse

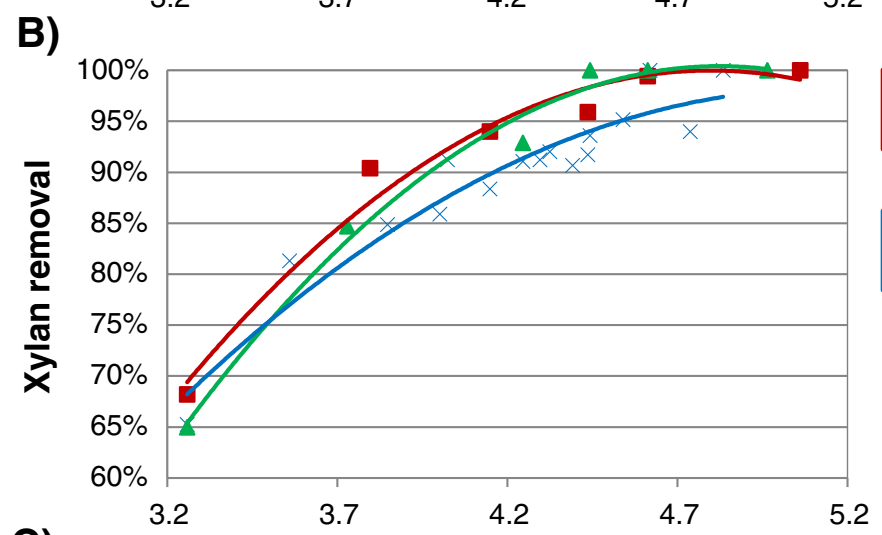

C)

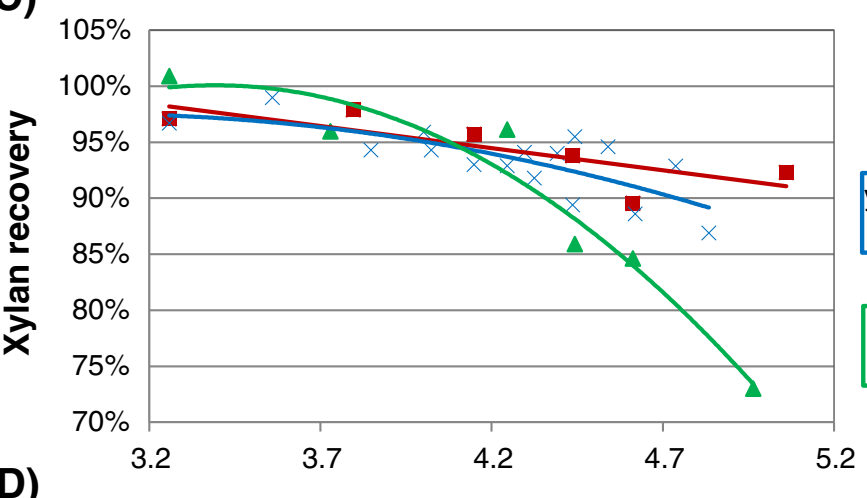

$y=-0.13 x^{2}+1.24 x-1.97$ $\mathrm{R}^{2}=0.97$

- Corn Stover

$y=-0.08 x^{2}+0.86 x-1.24$ $R^{2}=0.91$

\section{$\times$ Poplar}

$y=-0.14 x^{2}+1.38 x-2.32$ $R^{2}=0.99$

$\triangle$ Bagasse

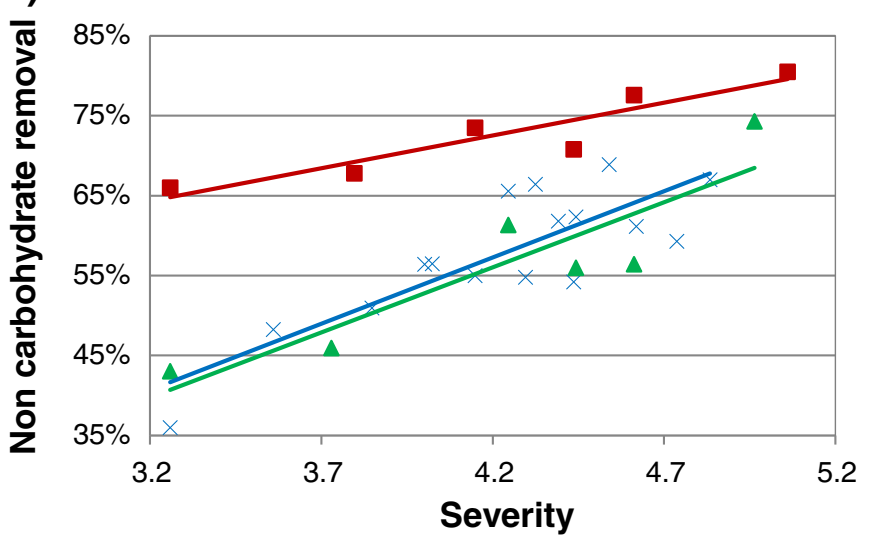

$y=-0.04 x+1.11$ $R^{2}=0.64$

- Corn Stover

$y=-0.03 x^{2}+0.15 x+0.75$ $R^{2}=0.56$

$\times$ Poplar $y=-0.11 x^{2}+0.73 x-0.24$ $R^{2}=0.94$

$\triangle$ Bagasse

$y=0.08 x+0.38$ $\mathrm{R}^{2}=0.86$

- Corn Stover

$y=0.17 x-0.12$ $\mathrm{R}^{2}=0.70$

$\times$ Poplar

$y=0.16 x-0.12$ $\mathrm{R}^{2}=0.81$

\section{$\triangle$ Bagasse}

Figure 3 Comparison between poplar, corn stover and bagasse. Glucan conversion after $96 \mathrm{~h}(\mathbf{A}), \mathrm{XMG}$ removal (B), XMG recovery (C) and non-carbohydrate removal (D) against severity of pretreatment. 
obtained for poplar, as illustrated in Figure 3B. Removal of XMG increased from $65 \%$ at a severity of $3.2(8 \mathrm{~min}$, $\left.180^{\circ} \mathrm{C}, \mathrm{R}_{\mathrm{L} / \mathrm{S}}=0.25\right)$ to $100 \%$ at a severity of $4.4-4.6(16-$ $\left.24 \mathrm{~min}, 210^{\circ} \mathrm{C}, \mathrm{R}_{\mathrm{L} / \mathrm{S}}=0.125-0.083\right)$. Since glucan conversion exhibits much more variability than XMG removal among the three feedstocks tested, factors other than XMG removal must affect the amenability to enzymatic attack. XMG recovery, shown in Figure 3C, is above 95\% for all feedstocks at severities below 4.1 but decreases above a severity of 4.1 due to degradation. Degradation products data are provided in Additional file 1. The recovery decreases fastest for bagasse, indicating that XMG in bagasse is the most susceptible to degradation among the substrates tested due to chemical and morphological differences.

\section{Extraction of non-carbohydrate carbon}

Removal of non-carbohydrate carbon was evaluated by evaluating the difference between total dry weight and carbohydrate content (anhydrous basis) after and before pretreatment. Removal of non-carbohydrate carbon, illustrated in Figure 3D, is not statistically different for poplar and bagasse, but is much higher for corn stover. It increases from about $40 \%$ to about $70 \%$ for poplar and bagasse and from $65 \%$ to $80 \%$ for corn stover at severities of 3.2 to about 4.9. This difference is a potential explanation for the higher amenability to SSF of corn stover pretreated at severities below 4.7 (Figure 1a and $4 \mathrm{a})$, since lignin has been shown to impede glucan conversion $[14,22]$.

\section{Optimization of FT pretreatment conditions on poplar}

Central composite design was used to generate response surfaces for various performance metrics as a function of temperature and reaction time for milled poplar. Glucan conversion, XMG removal, and non-carbohydrate removal were evaluated. The response surfaces, shown in Figure 4, were fit to a quadratic model. The adjusted coefficient of determination (adjusted $\mathrm{R}^{2}$ ) and the $\mathrm{p}$-value for the F-tests were used to evaluate the validity of the model and are provided in Additional file 2: Table S1 in supplemental materials. Additional file 2: Table S1 also presents the parameters of the quadratic model equations. All the models presented in this paper have $\mathrm{p}$-values much smaller than 0.0003 , indicating the models are statistically significant. The adjusted $\mathrm{R}^{2}$ values range from 0.76 to 0.95 . The points marked on the contour plots represent data points (Additional file 3: Table S2 in supplemental materials).

As shown in Figure 4a, the model predicts that a maximum glucan solubilization of about $86 \%$ occurs at $210^{\circ} \mathrm{C}$ and $16 \min \left(R_{L / S}=0.125\right)$. The XMG solubilized and recovered in the FT hydrolyzate is 5 to $15 \%$ lower than XMG removal, consistent with XMG degradation.
Complete removal of XMG is beneficial for the fermentation of the pretreated solids, but degradation is not desired [5]. Thus, an optimal point was found at $24 \mathrm{~min}$ and $210^{\circ} \mathrm{C}$ $\left(\mathrm{R}_{\mathrm{L} / \mathrm{S}}=0.083\right)$ where all of the XMG is removed from the solid and $89 \%$ of the XMG was recovered in the hydrolyzate. A distinguishing feature of FT pretreatment is the high degree of solubilization of non-carbohydrate carbon. In particular, non-carbohydrate removal was $65 \%$ for pretreatment of $24 \mathrm{~min}$ or more at temperatures at or above $200^{\circ} \mathrm{C}$.

\section{Exploration of conversion systems}

Comparison of alternative conversion systems is of obvious interest, and has not been undertaken previously on milled FT-pretreated cellulosic feedstocks. C. thermocellum and SSF were compared on FT pretreated poplar (FTP), ball milled FT pretreated poplar (BMFTP), and Avicel, a laboratory microcrystalline cellulose.

Under the conditions tested, which are intended for intrinsic comparison and are not representative of an industrial process, $C$. thermocellum solubilized all substrates tested more rapidly and more completely as compared to SSF (Figure 5). In particular, solubilization of FTP by C. thermocellum after 4 days (93\%) was $32 \%$ higher than by $\mathrm{SSF}$ at $8.4 \mathrm{mg}$ cellulase/g glucan (5 FPU/g glucan). Ball milling prior to hydrolysis substantially accelerated hydrolysis for both SSF and C. thermocellum, with C. thermocellum demonstrating higher rate and yield. In particular, $C$. thermocellum solubilized $98 \%$ of the cellulose in BMFTP in 2 days whereas SSF at 5 FPU/glucan required 5 days to achieve $88 \%$ solubilization.

\section{Conclusions}

Hot water FT pretreatment provides highly digestible solids and high sugar recovery for various types of milled biomass. For example, $90 \%$ of the glucan in bagasse was converted after $96 \mathrm{~h}$ of SSF and 84\% of the XMG was recovered when FT pretreated for $12 \mathrm{~min}$ at $220^{\circ} \mathrm{C}$ $\left(\mathrm{R}_{\mathrm{L} / \mathrm{S}}=0.167\right)$ versus $68 \%$ glucan conversion and $69 \%$ XMG recovery when batch pretreated for $14 \mathrm{~min}$ at the same temperature. It was found that the optimal reaction times and temperatures for FT pretreatment are 16 $\min$ at $200^{\circ} \mathrm{C}\left(\mathrm{R}_{\mathrm{L} / \mathrm{S}}=0.125\right)$ for corn stover, $16 \mathrm{~min}$ at $210^{\circ} \mathrm{C}\left(\mathrm{R}_{\mathrm{L} / \mathrm{S}}=0.125\right)$ for poplar and $12 \mathrm{~min}$ at $220^{\circ} \mathrm{C}\left(\mathrm{R}_{\mathrm{L} /}\right.$ $s=0.167)$ for bagasse. At those conditions, the glucan conversion after $96 \mathrm{~h}$ in SSF was 93\% for corn stover, $86 \%$ for poplar and $90 \%$ for bagasse and the XMG recovery was $96 \%$ for corn stover, $97 \%$ for poplar and $85 \%$ for bagasse. Thus, corn stover gives high glucan conversion yields at substantially lower severity than poplar or bagasse. XMG removal is rather similar although perhaps a bit less for poplar. The fact that glucan conversion is more different than XMG removal suggests that factors in addition to XMG removal impact amenability of 


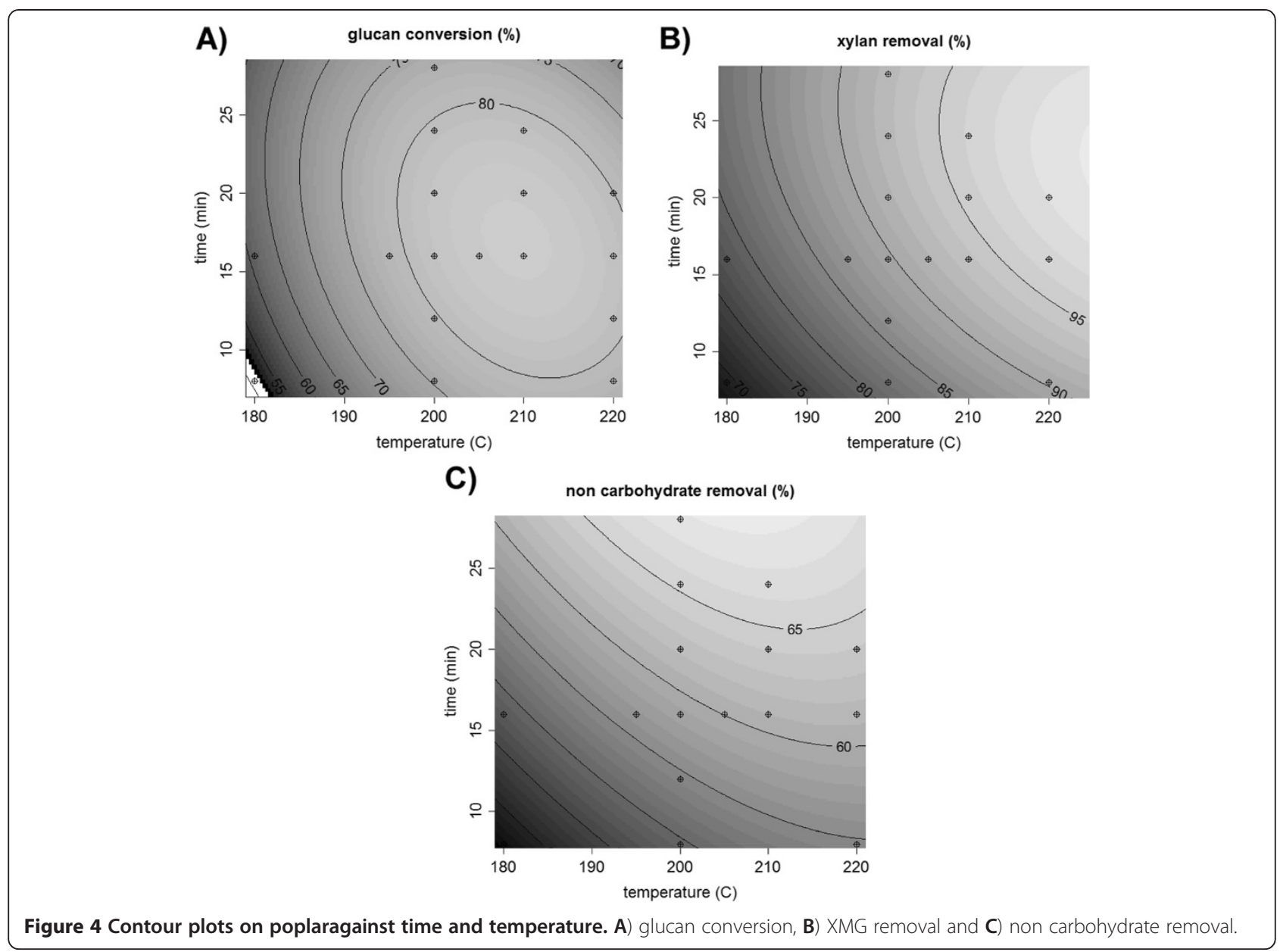

glucan to enzymatic attack. Higher removal of noncarbohydrate carbon was observed for corn stover than for poplar and bagasse, which may contribute to the lower severity required for corn stover pretreatment. XMG recovery is above $90 \%$ for all substrates below $210^{\circ} \mathrm{C}$ but is notably lower for bagasse at the high severities required to achieve high yields. C. thermocellum converts glucan more rapidly and completely than SSF under the conditions tested on Avicel and FT pretreated poplar. For example, after 4 days, C. thermocellum conversion of FT pretreated poplar was 32\% higher than SSF.

\section{Methods}

\section{Materials}

Poplar (Populus tremuloides) obtained from Meriden, $\mathrm{NH}$ was harvested in the summer and 1 to 4 " diameter trunks and branches were chipped $(\sim 1 / 2$ inch largest dimension). The chips were allowed to dry to a moisture content of $7 \%$ in ambient air at room temperature. Wet poplar was never dried and its moisture content was measured to be $50 \%$. Corn stover used for the CAFI project originally supplied by BioMassAgriProducts (BMAP,
Harlan, IA) [8] was kindly provided by Dr. Bruce Dale's lab at Michigan State University. Sugarcane from which bagasse was produced was harvested fresh in the winter from central Florida was provided by Mascoma Corporation (Lebanon, NH). All feedstocks were knife-milled (Model 3379 K35, Thomas Scientific, Swedesboro, NJ) to pass through a $2 \mathrm{~mm}$ screen. Bagasse was also sieved to remove dust and particles smaller than $105 \mu \mathrm{m}$. Flowthrough pretreated poplar was ball-milled for $60 \mathrm{~min}$ (Model no. SFM-3, MTI Corporation, Richmond, CA) when noted in the text. The composition of representative samples, shown in Table 1, was determined according to the NREL Laboratory Analytical Procedures (LAP's) [24]. Avicel PH 105 was purchased from FMC Corporation (Philadelphia, PA). Spezyme CP cellulase was kindly provided by Genencor International Inc. (Rochester, NY) and Novozyme188 $\beta$-glucosidase was purchased from Sigma-Aldrich (St. Louis, MO). All samples were refrigerated at $4^{\circ} \mathrm{C}$.

\section{FT apparatus and experiments}

The FT experiments followed a procedure similar to that described previously by Liu and Wyman $[19,20]$. The 

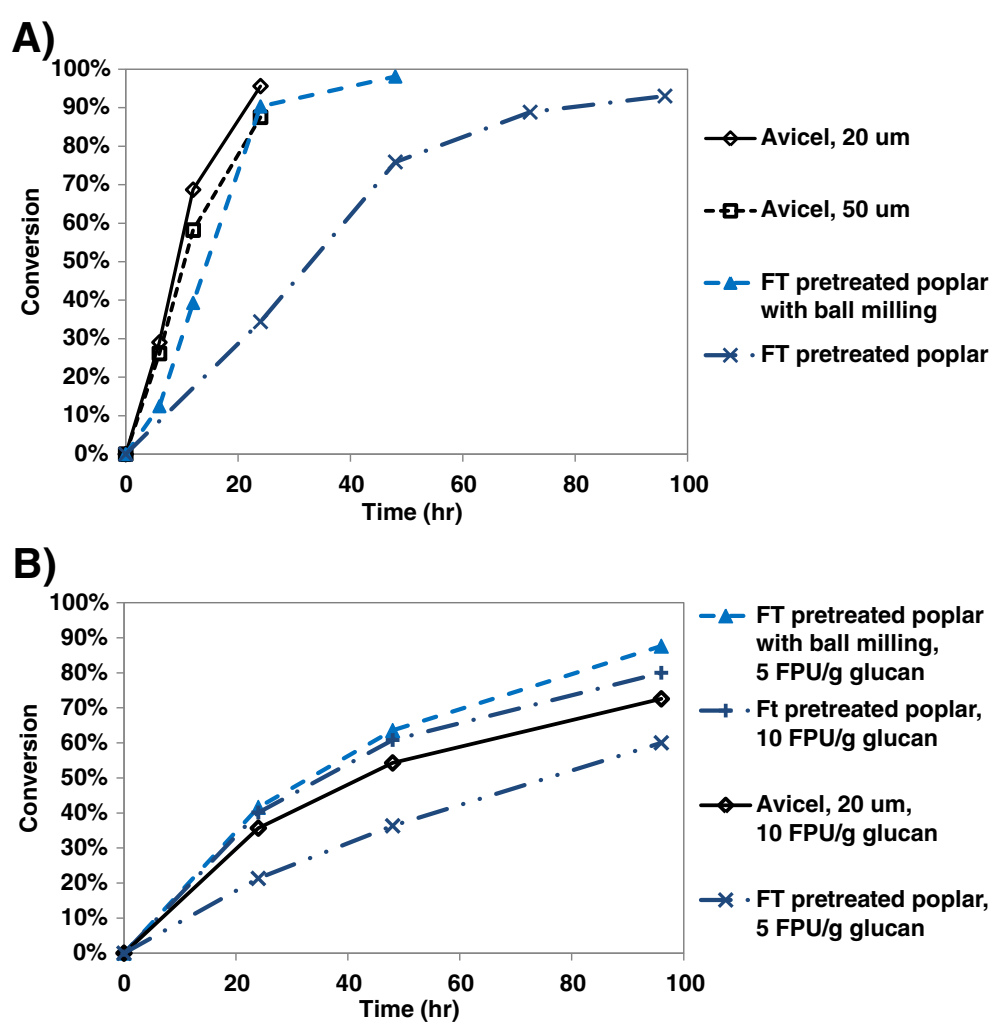

Figure 5 Glucan conversion on various substrates for A) C. thermocellum and B) simultaneous saccharification and fermentation (SSF).

reactor was a $16 \mathrm{~cm}$ long stainless steel tube with an internal diameter of $2.1 \mathrm{~cm}$, corresponding to a volume of $56 \mathrm{~mL}$. Filter gaskets with $20 \mu \mathrm{m}$ pore size, kindly provided by Mott Corporation (Farmington, CT), were used at the inlet and outlet of the tube reactor to contain the solids. All tubes and fittings were stainless steel $316 \mathrm{~L}$ purchased from Swagelok (Bangor, Maine). 12.6 $\pm 0.5 \mathrm{~g}$ of feedstock was loaded in the reactor $(22.5 \mathrm{w} / \mathrm{v} \%)$ Water was pumped through the reactor using a Lab Alliance dual piston pump (Prep 100, Scientific Systems, PA) at $30 \mathrm{~mL} / \mathrm{min}$ at room temperature to wet the solids. Once the outlet liquid was devoid of air bubbles, the heating coil and reactor were lowered into a fluidized sand bath controlled at the desired temperature. The start of the reaction time was set arbitrarily as the time when the reactor was lowered into the sand bath and the heating

Table 1 Feedstock composition before pretreatment with the standard deviation on duplicates

\begin{tabular}{lllll}
\hline Feedstock & \%glucan & \%xylan & \%arabinan & \%lignin \\
\hline Corn Stover & $33.2 \pm 1.6$ & $23.2 \pm 0.4$ & $2.7 \pm 0.04$ & $17.2[11]$ \\
Bagasse & $40.4 \pm 1.3$ & $23.6 \pm 0.6$ & $1.5 \pm 0.1$ & $20.9 \pm 1.9$ \\
Popular & $37.8 \pm 0.5$ & $16.1 \pm 1.3$ & $0.9 \pm 0.2$ & $21.9 \pm 0.5$ \\
\hline
\end{tabular}

time was observed to be about $5 \mathrm{~min}$ by monitoring the temperature of the outlet water with a thermocouple. When the target reaction time was reached, the reactor was immersed in an ice water bath to quench the reaction. The water flow was stopped when the temperature at the outlet of the reactor dropped below $60^{\circ} \mathrm{C}$. Pretreatment times were varied from $8 \mathrm{~min}$ to $28 \mathrm{~min}$ and reaction temperatures ranged from $180^{\circ} \mathrm{C}$ to $225^{\circ} \mathrm{C}$. A triplicate was performed at $200^{\circ} \mathrm{C}$ for $12 \mathrm{~min}$ to estimate the error of replicate measurements.

\section{Batch experiments}

The apparatus described above was also used for pretreatments without flow. The procedure was modified from most batch pretreatments reported in the literature [22]. The modified batch pretreatment was conducted with the same conditions as the FT pretreatment except that water flow was stopped once the wetting water was devoid of air bubbles. The heating time was experimentally observed to be about $7 \mathrm{~min}$ in separate experiments where a thermocouple was inserted in the center of the reactor. An extra two minutes was allowed for batch compared to FT pretreatment to ensure equivalent reaction times at the target temperature. For example, reaction time reported as $12 \mathrm{~min}$ means $12 \mathrm{~min}$ for FT and 
14 min for batch. After a preset reaction time, the reactor immersed in an ice water bath for about $3 \mathrm{~min}$ until the reactor cooled to approximately $60^{\circ} \mathrm{C}$ and then the water flow was started again to collect the hydrolysate fraction of the pretreated mixture.

\section{Composition analysis}

Compositional analysis of the solid and liquid fractions was determined using NREL Laboratory Analytical Procedures (LAP's) [24]. Carbohydrates were analyzed via refractive index using an Aminex HPX-87 $\mathrm{H}$ column at $65^{\circ} \mathrm{C}$ on a Waters HPLC system (2695 Separations Module, Waters Corporation, Milford MA). Degradation products were analyzed via UV spectra using an Agilent Eclipse XD8-C18 column on a Thermo-Spectra System HPLC. Sugar recovery, XMG solubilization, XMG removal, non carbohydrate removal and glucan conversion (x) were calculated using equations $1,2,3,4$ and 5, respectively. The XMG solubilization in this paper corresponds to the XMG solubilized and recovered in the hydrolysate. Non carbohydrate removal is calculated using the concentration of carbohydrates in each solids sample (equation 4), which includes in this study glucose, xylose and arabinose. Mannose and galactose were measured once for each substrate and each was found to be less than one percent.

$$
\begin{aligned}
& \text { sugar recovery }(\%)= \\
& \begin{array}{c}
(\text { Sugar Weight })_{\text {hydrolyzate }}+(\text { Sugar Weight })_{\text {final }} \\
(\text { Sugar Weight })_{\text {initial }}
\end{array} \times 100 \\
& X M G \text { solubilisation }(\%)=\frac{(X M G \text { Weight })_{\text {hydrolyzate }}}{(X M G \text { Weight })_{\text {initial }}} \\
& \times 100 \\
& \times M G(\%)=\frac{(X M G \text { Weight })_{\text {initial }}-(X M G \text { Weight })_{\text {final }}}{(X M G \text { Weight })_{\text {initial }}} \\
& \times 100
\end{aligned}
$$

$$
x=\frac{(\text { Glucan Weight })_{\text {initial }}-(\text { Glucan Weight })_{\text {final }}}{(\text { Glucan Weight })_{\text {initial }}}
$$

\section{SSF}

SSF was carried out using a protocol similar to that described previously [25] with $20 \mathrm{~g} / \mathrm{L}$ initial glucan loading for Figures 1, 2, 3, and 4 and $5 \mathrm{~g} / \mathrm{L}$ initial glucan loading for Figure 5 for comparison with $C$. thermocellum. $5 \%(\mathrm{v} / \mathrm{v})$ inoculation of Saccharomyces cerevisae strain D5A (NREL) was prepared via overnight culture for $16 \mathrm{~h}$ in YPD media (Sigma Y1375). The experiments were performed in $125 \mathrm{~mL}$ serum bottles (Bellco, Vineland, NJ), which were prefilled with the solid residue after pretreatment and media, sealed and purged with nitrogen. The bottles were sterilized by autoclaving at $121^{\circ} \mathrm{C}$ for $45 \mathrm{~min}$ and brought to room temperature prior to addition of enzymes and yeast. The Spezyme CP cellulase, assumed to contain 1 FPU per $0.6 \mathrm{mg}$ of protein, was loaded at 5 or $10 \mathrm{FPU} / \mathrm{g}$ glucan, as noted in the text. It was supplemented by Novozyme188 $\beta$-glucosidase at an activity ratio of 3 IU per FPU. The medium used was developed by Kadam and Newman [26] and consists of $0.3 \%(\mathrm{v} / \mathrm{v})$ corn steep liquor supplemented by $5 \mathrm{mM} \mathrm{MgSO}$. The inoculum was prepared from frozen stock in YPD media. The inoculated serum bottles were incubated at $37^{\circ} \mathrm{C}$ with $200 \mathrm{rpm}$ shaking for the duration of the experiment. The remaining solids were processed and analyzed according to the composition analysis described above after 24 and $96 \mathrm{~h}$.

\section{C. thermocellum fermentation}

C. thermocellum fermentation was carried out with 5 $\mathrm{g} / \mathrm{L}$ initial glucan loading and $10 \%(\mathrm{v} / \mathrm{v})$ inoculation of C. thermocellum ATCC 27405 (American Type Culture Collection, Manassas, VA). The experiments were performed in $125 \mathrm{~mL}$ serum bottles (Bellco, Vineland, NJ), which were prefilled with substrate and media, sealed and purged with nitrogen. The bottles were sterilized by autoclaving at $121^{\circ} \mathrm{C}$ for $45 \mathrm{~min}$ and then brought to room temperature before adding $\mathrm{A}, \mathrm{B}, \mathrm{C}, \mathrm{D}, \mathrm{E}$ and $\mathrm{F}$ solutions as described by Shao et al. [25]. The medium was prepared according to Zhang and Lynd [27] using chemically-defined media for thermophilic clostridia

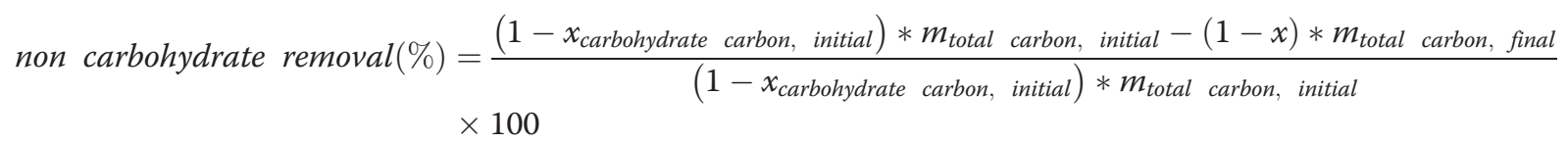


(MTC). The inoculum was prepared from frozen stock of a single isolated colony on $5 \mathrm{~g} / \mathrm{L}$ Avicel $\mathrm{PH} 105$ in MTC media. The inoculated serum bottles were placed in a $55^{\circ} \mathrm{C}$ shaking incubator (New Brunswick Scientific, Inova 4080) at $200 \mathrm{rpm}$ for the duration of the experiment. Sample collection and processing were the same as described for SSF.

\section{Additional files}

Additional file 1: Figure S1. Measured degradation products in the hydrolysate for FT corn stover, bagasse and poplar. Provides degradation products data explanatory for a decrease in sugar recovery.

Additional file 2: Table S1. Quadratic models for FT pretreatment on poplar at various times and temperatures. Provides the mathematical model, the adjusted $R^{2}$ value and the $p$-value for Figure 4 .

Additional file 3: Table S2. Compositional mass balances on pretreatment and SSF. Provides supporting data in grams for all Figures. Initial glucan is $1 \mathrm{~g}$ for Figures 1, 2, 3, and 4 and $0.25 \mathrm{~g}$ for Figure 5. SSF glucan residue is reported after 4 days.

\section{Competing interests}

Lee R. Lynd is a cofounder and share-holder of Mascoma Corporation, who may have a financial interest in the current study.

\section{Author's contributions}

VA performed experiments for optimizing pretreatment conditions and for comparing feedstocks, as well as drafted the paper. XS performed the experiments for comparing conversion systems. All authors participated in designing the study, have revised the paper critically for intellectual content and have read and approved the final manuscript.

\section{Acknowledgements}

The authors are grateful for the support provided by funding grants from the BioEnergy Science Center (BESC), a U.S. Department of Energy (DOE) Research Center supported by the Office of Biological and Environmental Research in the DOE Office of Science, Oak Ridge National Laboratory, and Mascoma Corporation. Oak Ridge National Laboratory is managed by University of Tennessee UT-Battelle LLC for the Department of Energy under Contract No. DE-AC05-000R22725. We thank Dr. Mark Borsuk at the Dartmouth College for help on design of experiments and statistical analysis, Walter E. Scott for his help in performing analytical procedures, Phil Thorne for his help in analyzing degradation products and Dave Hogsett for critically reviewing this paper.

\section{Author details}

${ }^{1}$ Dartmouth College, Hanover, NH 03755, USA. ²DOE BioEnergy Science Center, Oak Ridge National Laboratory, Oak Ridge, TN 37831, USA. ${ }^{3}$ Mascoma Corporation, NH 03766, Lebanon, USA.

Received: 25 March 2012 Accepted: 2 July 2012

Published: 19 July 2012

\section{References}

1. Lynd L, Cruz CHB: Make way for ethanol. Science 2010, 330:1176-1177.

2. Nathanael G, Celik FE, Dale B, Jackson M, Jayawardhana $K$, Jin H, Larson ED, Laser MS, Lynd LR, MacKenzie D, Mark J, McBride J, McLaughlin S, Saccardi D: Growing energy: how biofuels can help end America's oil dependence. Natural Resources Defense Council 2004. http://www.nrdc.org/ air/energy/biofuels/biofuels.pdf

3. Farrell A, Plevin RJ, Turner BT, Jones AD, O'Hare M, Kammen DM: Ethanol can contribute to energy and environmental goals. Science 2006, 311:506-508.

4. Lynd L, Laser MS, Bransby D, Dale BE, Davison B, Hamilton R, Himmel M, Keller M, McMillan JD, Sheehan J, Wyman CE: How biotech can transform biofuels. Nat Biotechnol 2008, 26:169-172.
5. Alvira P, Tomás-Pejó E, Ballesteros E, Negro MJ: Pretreatment technologies for an efficient bioethanol production process based on enzymatic hydrolysis: A review. Bioresour Technol 2010, 101:4851-4861.

6. Himmel M, Ding SY, Johnson DK, Adney WS, Nimlos MR, Brady JW, Foust TD: Biomass Recalcitrance: Engineering Plants and Enzymes for Biofuels Production. Science 2007, 315:804-807.

7. McMillan J, Himmel ME, Baker JO, Overend RP: Pretreatment of lignocellulosic biomass. DC: Washington; 1994

8. Wyman C, Dale B, Elander RT, Holtzapple M, Ladisch MR, Lee YY: Coordinated development of leading biomass pretreatment technologies. Bioresour Technol 2005, 96:1959-1966.

9. Yang B, Wyman CE: Pretreatment: the key to unlocking low-cost cellulosic ethanol. Biofuels, Bioprod Biorefin 2008, 2:26-40.

10. Mosier N, Wyman CE, Dale BE, Elander R, Lee YY, Holtzapple M, Ladisch M: Features of promising technologies for pretreatment of lignocellulosic biomass. Bioresour Technol 2005, 96:673-686.

11. Olson D, McBride JE, Shaw AJ, Lynd LR: Recent progress in consolidated bioprocessing. Curr Opin Biotechnol 2011, 23:396-405.

12. Zhang Y-H, Himmel ME, Mielenz JR: Outlook for cellulase improvement: screening and selection strategies. Biotechnol Adv 2006, 24:452-481.

13. Simmons B, Loque D, Blanch HW: Next-generation biomass feedstocks for biofuel production. Genome Biol 2008, 9:242.

14. Yang B, Gray MC, Liu C, Lloyd TA, Stuhler SL, Converse A, Wyman CE: Unconventional relationships for hemicellulose hydrolysis and subsequent cellulose digestion. In ACS Symposium Series on Lignocellulose Biodegradation. Edited by Saha BS, Hayashi K. Washington: American Chemical Society; 2004:100-125.

15. Overned $R$, Chornet E: Fractionation of lignocellulosics by steam-aqueous pretreatments. Philos Trans R Soc London Set A 1987, 321:523-536.

16. Walsum G, Allen SG, Spencer MJ, Laser MS, Antal MJ Jr, Lynd LR: Conversion of lignocellulosics pretreated with liquid hot water to ethanol. Appl Biochem Biotechnol 1996, 57-58:157-170.

17. Wooley R, Ruth M, Glassner D, Sheehan J: Process design and costing of bioethanol technology: a tool for determining the status and direction of research and development. Biotechnol Prog 1999, 15:794-803.

18. Lynd L, Elander RT, Wyman CE: Likely features and costs of mature biomass ethanol technology. App/ Biochem Biotechnol 1996, 57/58:741-761.

19. Liu C, Wyman CE: The effect of flow rate of compressed hot water on xylan, lignin and total mass removal from corn stover. Ind Eng Chem Res 2003, 5409:5416.

20. Liu C, Wyman CE: Impact of fluid velocity on hot water only pretreatment of corn stover in a flowthrough reactor. Appl Biochem Biotechnol 2004, 113-116:113-116.

21. Liu C, Wyman CE: The effect of flow rate of very dilute sulfuric acid on xylan, lignin, and total mass removal from corn stover. Ind Eng Chem Res 2004, 43:2781-2788.

22. Yang B, Wyman CE: Effect of xylan and lignin removal by batch and flowthrough pretreatment on the enzymatic digestibility of corn stover cellulose. Biotechnol Bioeng 2004, 86:88-95.

23. Yang B, Wyman CE: Characterization of the degree of polymerization of xylooligomers produced by flowthrough hydrolysis of pure xylan and corn stover with water. Bioresour Technol 2008, 99:5756-5762.

24. National Renewable Energy Laboratory: Standard biomass analytical procedures. http://www.nrel.gov/biomass/analytical_procedures.html.

25. Shao X, Jin M, Guseva A, Liu C, Balan V, Hogsett D, Dale BE, Lynd LR: Conversion for Avicel and AFEX pretreated corn sover by Clostridium thermocellum and simultaneous saccharification and fermentation: insights into microbial conversion of pretreated cellulosic biomass. Bioresour Technol 2011, 102:8040-8045.

26. Kadam K, Newman MM: Development of a low-cost fermentation medium for ethanol production from biomass. Appl Microbiol Biotechnol 1997, 47:625-629.

27. Zhang Y, Lynd LR: Quantification of cell and cellulase mass concentrations during anaerobic cellulose fermentation: development of an enzyme-linked immunosorbent assay-based method with application to Clostridium thermocellum batch cultures. Anal Chem 2003, 75:219-227.

doi:10.1186/1754-6834-5-49

Cite this article as: Archambault-Leger et al:: Integrated analysis of hydrothermal flow through pretreatment. Biotechnology for Biofuels 2012 5:49. 